\title{
Circulating Long Noncoding RNA HOTAIR is an Essential Mediator of Acute Myocardial Infarction
}

\author{
Lu Gao Yuan Liu Sen Guo Rui Yao Leiming Wu Lili Xiao Zheng Wang \\ Yuzhou Liu Yanzhou Zhang \\ Department of Cardiology, the First Affiliated Hospital of Zhengzhou University, Zhengzhou, China
}

\section{Key Words}

Acute myocardial infarction • Long noncoding RNAs • HOTAIR • Biomarkers • MiR-1

\begin{abstract}
Background/Aims: Acute myocardial infarction (AMI) is one of the leading causes of death in the world. However, specific diagnostic biomarkers have not been fully determined, and candidate regulatory targets for AMI have not been identified to date. Long noncoding RNAs (IncRNAs) are a class of RNA molecules that have diverse regulatory functions during embryonic development, normal life, and disease in higher organisms. However, research on the role of IncRNAs in cardiovascular diseases, particularly AMI, is still in its infancy. HOX antisense intergenic RNA (HOTAIR), a $2.2 \mathrm{~kb}$ IncRNA, was initially described as a modulator of HOX gene expression. Recent studies have illustrated the important role of HOTAIR in cancer progression, but few studies have reported its function in cardiac disease, including AMI. In the current study, we aimed to detect the expression of HOTAIR during AMI and to explore its function in hypoxia-induced cardiomyocyte injury in neonatal cardiomyocytes. Methods: In 50 consecutively enrolled AMI patients, we examined the serum expression levels of HOTAIR and analysed its correlation with cardiac troponin I (cTnI) expression. Another 50 age- and sex-matched subjects served as healthy controls. Next, the HOTAIR expression was detected in the serum from C57BL/6J mice subjected to coronary artery ligation and in neonatal rat cardiomyocytes induced by hypoxia. Cultured cardiomyocytes apoptosis were measured by terminal deoxynucleotide transferase dUTP nick end labelling (TUNEL) staining. A search for miRNAs that had complementary base paring with HOTAIR was performed utilizing an online software program, and the interaction between miR-1 and HOTAIR was examined using a luciferase reporter assay. Results: Our study revealed that HOTAIR expression was significantly decreased in the serum of AMI patients compared with that of the healthy controls. Similarly, we observed that HOTAIR was downregulated in the serum of mice subjected to coronary artery ligation and in cultured cardiomyocytes exposed to hypoxia. Furthermore, we observed that the adenovirus vector-driven overexpression of HOTAIR dramatically limited hypoxiainduced myocyte apoptosis, whereas knockdown HOTAIR by AdshHOTAIR (adenoviral short hairpin HOTAIR) exhibited the opposite phenotype. Mechanistically, we discovered that the L. Gao, Y. Liu and S. Guo are co-first authors.
\end{abstract}

Yanzhou Zhang

KARGER
Department of Cardiology, The First Affiliated Hospital of Zhengzhou University, No.1 Jianshe East Road, Zhengzhou (China)

Tel. +86 13676918168, E-Mail yzzhang6@163.com 
cardioprotective function of HOTAIR is partly based on the negative regulation of miR-1. Conclusions: Taken together, the results of our study suggest that HOTAIR is a protective factor for cardiomyocytes and that the plasma concentration of HOTAIR may serve as a biomarker for human AMI diagnosis.

(C) 2017 The Author(s)

Published by S. Karger AG, Basel

\section{Introduction}

Acute myocardial infarction (AMI) is a severe cardiovascular disease and a serious threat to human life [1]. AMI can cause congestive heart failure and malignant arrhythmia leading to high morbidity and mortality [2,3]. While thrombolysis and percutaneous coronary intervention (PCI) can significantly improve the prognosis for patients with AMI, there are still many patients with AMI that eventually develop heart failure or arrhythmia due to the unclear aetiology of AMI [4]. Thus, in order to provide valuable benefits to current therapies, it is necessary to distinguish novel, highly sensitive and specific biomarkers for the early diagnosis of AMI and to identify possible regulatory targets of AMI.

Among noncoding RNAs, short noncoding RNAs, such as microRNAs, have been extensively investigated as potential disease biomarkers and therapeutic targets [57]. Unlike microRNAs, a novel class of noncoding RNAs, longer than 200 nucleotides and termed long noncoding RNAs (lncRNAs), has recently emerged [8, 9]. As research has progressed, thousands of IncRNAs have been confirmed to be involved in various biological processes, including cell growth, differentiation, cell proliferation and apoptosis [10-13]. In addition, findings from recent studies suggest that circulating lncRNAs are useful markers for the diagnosis of multiple cancers. In cardiovascular disease, the profiles of IncRNAs in plasma and serum are altered, suggesting a variety of opportunities for the development of circulating lncRNAs as blood-based markers for molecular diagnostics. For example, several lncRNAs have been explored as prognostic biomarkers for heart failure $[14,15]$. In addition, several lncRNAs have been found to be regulated in heart tissue during cardiac hypertrophy $[16,17]$. However, studies about the use of circulating lncRNAs as biomarkers of AMI are still in their infancy.

The IncRNA HOX antisense non-coding RNA (HOTAIR), a 2.2 kb lncRNA transcribed from the HOXC locus, was initially described to downregulate HOXD gene expression through the recruitment of polycomb repression complex 2 (PRC2) to trimethylate histone H3 lysine 27 (H3K27) [18-20]. So far, HOTAIR has been proven to play an important role in the development and progression of various complicated diseases, including breast cancer, colon cancer, oesophageal squamous cell carcinoma and cardiac disease. In addition, HOTAIR has been shown to be regulated by hypoxia and to modulate cell proliferation, apoptosis, migration and invasion $[21,22]$. The role of HOTAIR in the cardiac system has been gradually recognized, HOTAIR was reported as being significantly upregulated in cardiomyocytes from septic mice and as being downregulated in a mouse model of cardiac hypertrophy, HOTAIR was also found downregulated in heart failure patients from RNA-seq data [23-26]. However, until now, the expression level and role of HOTAIR in other cardiovascular diseases have been unexplored. In this study, we aimed to determine the level of plasma HOTAIR in patients with AMI, which can be used to detect and monitor myocardial injury, and to explore the functions of HOTAIR in hypoxia-induced neonatal rat cardiomyocyte injury.

\section{Materials and Methods}

Reagents

Antibodies against the following proteins were purchased from Cell Signaling Technology: Bax (\#2772), Bcl-2 (\#2870), cleaved caspase-3 (\#9661), caspase-3 (\#9662), and GAPDH (\#2118). A BCA protein assay kit (\#23225) was purchased from Pierce (Thermo Scientific). Cell culture reagents and all other reagents were purchased from Life Technologies. 


\section{Cellular Physiology Cell Physiol Biochem 2017;44:1497-1508 \begin{tabular}{ll|l} 
and Biochemistry Published onIIne: December 01, 2017 & $\begin{array}{l}\text { C) } 2017 \text { The Author(s). Published by S. Karger AG, Basel } \\
\text { www.karger.com/cpb }\end{array}$ \\
\hline
\end{tabular}}

Gao et al.: HOTAIR Modulates Acute Myocardial Infarction

\section{Patients and Ethics Statement}

The cohort included 50 patients with AMI and 50 healthy volunteers. The inclusion criteria for AMI patients were based on the 2015 ESC/AHA/ACC guidelines. AMI was diagnosed based on a combination of several parameters: ischaemic symptoms, increased cardiac troponin I (cTnI) and creatine kinase-MB (CK$\mathrm{MB}$ ) expression and a pathological Q wave. All AMI patients were diagnosed for the first time and underwent primary PCI.

The protocols used in the present study were conducted according to the principles expressed in the Declaration of Helsinki and were approved by the Medical Ethics Committee of the First Affiliated Hospital of Zhengzhou University. Patients were admitted to the Department of Cardiology at the First Affiliated Hospital of Zhengzhou University between January 2015 and April 2016. In addition, written informed consent was obtained from all enrolled patients, and the data for clinical presentation/history, cardiovascular risk factors and medication records were also obtained.

\section{Sample collection and storage}

The initial blood sample (denoted as T0) was collected immediately after the AMI patient was admitted to the First Affiliated Hospital of Zhengzhou University. The 5 subsequent blood samples were obtained at 4,12, 24, 48, and 72 hours after the first collection and are denoted as $4 \mathrm{~h}, 12 \mathrm{~h}, 24 \mathrm{~h}, 48 \mathrm{~h}$ and $72 \mathrm{~h}$, respectively. Blood samples $(5 \mathrm{ml})$ from subjects were collected via direct venous puncture into tubes containing sodium citrate and centrifuged at $1000 \mathrm{~g}$ for $5 \mathrm{~min}$; then, the supernatant (plasma) was carefully extracted, aliquoted, transferred to RNase/DNase-free tubes and stored at $-80^{\circ} \mathrm{C}$ for the following experiments.

\section{Experimental animals and AMI mouse model}

All animal procedures were performed in accordance with the Guide for the Care and Use of Laboratory Animals published by the US National Institutes of Health and were approved by the Animal Care and Use Committee of the Department of Cardiology, Zhengzhou University. The male C57BL/6J mice used in the experiment were obtained from the Institute of Laboratory Animal Science, Chinese Academy of Medical Sciences (Beijing, China).

The left anterior descending (LAD) coronary artery ligation was performed as previously described [27]. Briefly, mice were anaesthetized with sodium pentobarbital (90 mg/kg, P3761, Sigma-Aldrich) by intraperitoneal injection and were ventilated with a small animal ventilator (model VFA-23-BV, Kent Scientific). The pericardium of each mouse was opened after thoracotomy, and a 7/0 silk suture was used to encircle and ligate the LAD. In sham operated mice, the left coronary artery was encircled without ligation. After full recovery, the animals were returned to independently ventilated cages. Blood samples were collected $0,1,6,12$, and 24 hours after the surgery was performed.

\section{Western blotting}

Cultured cardiomyocytes were lysed using RIPA buffer, and the protein concentration was determined with a BCA protein assay kit. Protein extracts $(20 \mu \mathrm{g})$ were used for SDS-PAGE, and the proteins were transferred onto a polyvinylidene fluoride membrane that was incubated with various primary antibodies overnight at $4^{\circ} \mathrm{C}$. After incubation with secondary antibody for 1 hour at room temperature, the membranes were treated with ECL reagents and visualized using a Fluor Chem E imager according to the manufacturer's instructions. The specific protein expression level was normalized to GAPDH expression on the same nitrocellulose membrane.

\section{Quantitative real-time PCR}

Total mRNA was extracted from the serum and primary cells using TRIzol reagent (Invitrogen), and cDNA was synthesized using oligo (dT) primers with the Transcriptor First Strand cDNA Synthesis Kit (Roche). The PCR amplicons were quantified using SYBR Green PCR Master Mix (Applied Biosystems). For measuring HOTAIR expression, $1 \mu \mathrm{g}$ of total RNA was reverse transcribed using MulV reverse transcriptase (TransGen, Cat. AT101-02) and random hexamer primers in a $20 \mu \mathrm{l}$ reaction volume, and U6 expression was used as the internal control. For measuring miR-1 expression, $1 \mu \mathrm{g}$ of total RNA from each sample was reverse transcribed using microRNA-specific primers and M-MLV reverse transcriptase (Promega, Madison, WI, USA), and U6 expression was used as the internal control. 
Cardiomyocyte culture and infection with recombinant adenoviral vectors

To silence or overexpress HOTAIR, we infected neonatal rat cardiomyocytes (NRCMs) with adenoviral short hairpin HOTAIR (AdshHOTAIR) or adenoviral HOTAIR (AdHOTAIR). Cardiac myocyte cultures were prepared as described in our previous studies with minor alterations [28]. Briefly, PBS containing 0.03\% trypsin and $0.04 \%$ collagenase type II was used to isolate the cardiomyocytes from 1- to 2-day-old Sprague Dawley rats. After removing fibroblasts by a differential attachment technique, the cardiomyocytes were seeded at a density of $1 \times 10^{6}$ cells per well into six-well culture plates coated with gelatine in plating medium consisting of DMEM/F12 supplemented with $20 \%$ foetal calf serum, BrdU and penicillin/streptomycin. These myocytes were then infected with AdHOTAIR and AdshHOTAIR for 24h.

\section{Hypoxia induction}

To induce hypoxia, cultured cardiomyocytes were incubated in a Biospherix C-Chamber inside a standard culture chamber. The oxygen $\left(\mathrm{O}_{2}\right)$ concentration was maintained at $5 \%$ inside the $\mathrm{C}$-Chamber, and the carbon dioxide $\left(\mathrm{CO}_{2}\right)$ concentration was maintained at $5 \%$ by mixing $\mathrm{N}_{2}$ and $\mathrm{CO}_{2}$ with a ProOx 110 oxygen controller and a $\mathrm{ProCO}_{2}$ controller. Meanwhile, the control group without hypoxia was maintained in a normal atmosphere of $5 \% \mathrm{CO}_{2}$ and $95 \%$ air at $37^{\circ} \mathrm{C}$.

\section{TUNEL assay}

Cultured cardiomyocyte apoptosis was measured by a terminal deoxynucleotide transferase dUTP nick end labelling (TUNEL) assay. Briefly, cardiomyocytes were cultured on coverslips in 6-well plates and were fixed in $4 \%$ paraformaldehyde. The TUNEL staining was performed using an in situ cell death detection kit (Minneapolis, MN, USA) according to the manufacturer's protocol. The number of TUNEL-positive cells and the number of total cells were counted under a fluorescence microscope.

\section{Luciferase Assay}

The predicted miR-1 binding site (HOTAIR-WT) and its mutant type (HOTAIR-MUT) were amplified and directly fused to the downstream of the luciferase reporter gene in the CMV promoter vector. In brief, cardiomyocytes grown in the 24-well plates were transfected with luciferase reporters and miR-1-mimic, or negative controls by using Fugene HD (Roche) according to the manufacturers' instruction for $24 \mathrm{~h}$. After that, cells were harvested and subjected to luciferase reporter assays using the kit from Promega and the ratios of firefly luciferase to Renilla luciferase were calculated with a Promega Glomax 2020 Single Tube Luminometer instrument (Promega) for each well.

\section{Statistical analysis}

All data are presented as the means \pm standard deviations (SDs). Student's two-tailed t-test was used to compare the means of two groups of samples, and two-way analysis of variance was used to compare more than two groups. The time course expression of the IncRNAs and cTnI were analysed using the repeatedmeasures ANOVA method, and the correlation between IncRNA and cTnI expression was analysed by using Pearson's correlation coefficient. All statistical analyses were accomplished by using SPSS 19.0 software, and $\mathrm{p}<0.05$ was considered statistically significant.

\section{Results}

\section{Pattern of plasma HOTAIR levels in the patients with AMI}

First, $50 \mathrm{AMI}$ patients and 50 healthy volunteers were selected to test whether circulating HOTAIR can serve as a novel biomarker of AMI. The baseline clinical characteristics of the cohort are shown in Table 1. There were no significant differences in age, sex, blood pressure, fasting glucose and other biochemical parameters between AMI patients and healthy volunteers. Six blood samples were obtained from each AMI patient at various time points (T0 h, $4 \mathrm{~h}, 12 \mathrm{~h}, 24 \mathrm{~h}, 48 \mathrm{~h}$, and $72 \mathrm{~h}$ ) to investigate the dynamic trend in circulating HOTAIR levels in the early phase of AMI. The first plasma sample was collected at $13.2 \pm 3.1$ hours after the onset of AMI symptoms (T0 h), and the other 5 time points were collected 4 , $12,24,48$ and $72 \mathrm{~h}$ after the T0 time point.

As shown in Fig. 1A, the expression level of HOTAIR in all AMI patients was significantly decreased in the early phase (the first 3 time points) following the occurrence of AMI 
compared with that of the healthy volunteers. In AMI patients, circulating HOTAIR was lowest from 6-12 $\mathrm{h}$ and showed a tendency to gradually return close to its control level over the next 3 days. The concentration of cTnI was simultaneously measured in the same AMI patient blood samples. Interestingly, the concentration of cTnI was remarkably increased in the early phase of AMI, peaked in 6-12 $\mathrm{h}$ and then gradually declined to near basal levels over the next 3 days (Fig. 1B). In addition, we detected a correlation between HOTAIR and cTnI, our results showed a negative correlation between the mRNA expression level of HOTAIR and cTnI concentration (Fig. 1C-D).

HOTAIR is reduced in the serum of AMI mice and in cultured cardiomyocytes exposed to hypoxia

To quantify the expression level of HOTAIR in the serum of AMI mice, we collected blood samples at $0,1,6$, and $24 \mathrm{~h}$ from left coronary artery ligation performed mice or the sham operated mice. As shown in Fig. 2A, quantitative RT-PCR results demonstrated that compared with the sham groups, AMI groups had a significantly downregulated expression level of HOTAIR at 1, 6, and $24 \mathrm{~h}$ after AMI $(0 \mathrm{~h})$. To detect the effect of hypoxia on HOTAIR expression in cultured cardiomyocytes, we collected RNA from cultured neonatal rat cardiomyocytes exposed to hypoxia for $0,1,6$, and $24 \mathrm{~h}$. As shown in Fig. 2B, quantitative RT-PCR showed that the expression level of HOTAIR was remarkably reduced in cardiomyocytes exposed to hypoxia for 1,6 , and $24 \mathrm{~h}$ compared with that of the normal control.

\section{HOTAIR is a myocardial-specific IncRNA}

Next, we wanted to determine whether HOTAIR is a myocardial-specific lncRNA and to evaluate the value of HOTAIR as a potential biomarker for myocardial injury. For this purpose, HOTAIR expression was detected in different the mice organs, including the heart, liver, spleen, lung, kidney, and muscle, using RT-PCR. The results suggested that HOTAIR is produced exclusively in the heart, and when we used the relative expression of HOTAIR in the heart as the standard, the expression levels of HOTAIR in other organs were significantly lower (Fig. 3A).

Table 1. Clinical characteristics of enrolled subjects. Results are expressed as median (interquartile range) or n (\%). BMI, body mass , DM, diabetes mellitus; SBP, systolic blood pressure; DBP, diHDL, high-density lipoprotein; LDL, low-density lipoprotein; CK-MB, creatine kinase-MB; cTnI, cardiac troponin I; ACEI/ARB, angiotensin converting enzyme inhibitor/angiotensin receptor blocker. Data are wn as the means $\pm \mathrm{SDs} ;{ }^{*} \mathrm{p}<0.01$ for the AMI groups vs. the control

\begin{tabular}{|c|c|c|c|}
\hline Variable & $\begin{array}{l}\text { AMI group } \\
(n=50)\end{array}$ & $\begin{array}{l}\text { Control group } \\
\qquad(\mathrm{n}=50)\end{array}$ & $P$ value \\
\hline Male/Female & $31 / 19$ & $28 / 22$ & 0.42 \\
\hline Age (years) & $61.2 \pm 11.3$ & $62.4 \pm 10.7$ & 0.46 \\
\hline BMI $\left(\mathrm{kg} / \mathrm{m}^{2}\right)$ & $24.3 \pm 2.6$ & $24.9 \pm 2.7$ & 0.53 \\
\hline \multicolumn{4}{|l|}{ Risk factors } \\
\hline Hypertension & $22(44 \%)$ & $23(46 \%)$ & 0.56 \\
\hline DM & $15(30 \%)$ & $12(24 \%)$ & 0.39 \\
\hline Dyslipidaema & $29(58 \%)$ & $28(56 \%)$ & 0.51 \\
\hline Smoking & $23(46 \%)$ & $21(42 \%)$ & 0.47 \\
\hline Heart rate (beats/minutes) & $72.2 \pm 13.4$ & $73.5 \pm 10.6$ & 0.44 \\
\hline SBP (mmHg) & $118.7 \pm 15.1$ & $115.8 \pm 14.2$ & 0.52 \\
\hline $\mathrm{DBP}(\mathrm{mmHg})$ & $79.5 \pm 11.8$ & $81.3 \pm 12.7$ & 0.48 \\
\hline $\mathrm{TC}(\mathrm{mmol} / \mathrm{L})$ & $4.22 \pm 0.69$ & $4.13 \pm 0.76$ & 0.41 \\
\hline $\mathrm{TG}(\mathrm{mmol} / \mathrm{L})$ & $1.69 \pm 0.63$ & $1.72 \pm 0.79$ & 0.39 \\
\hline HDL-C (mmol/L) & $1.23 \pm 0.87$ & $1.19 \pm 0.64$ & 0.45 \\
\hline LDL-C (mmol/L) & $2.62 \pm 1.03$ & $2.71 \pm 0.97$ & 0.37 \\
\hline CK-MB (IU/L) & $127.6 \pm 76.8$ & $7.45 \pm 3.26$ & $<0.01 *$ \\
\hline c-TnI (ng/ml) & $26.3 \pm 7.1$ & $0.03 \pm 0.01$ & $<0.01 *$ \\
\hline \multicolumn{4}{|l|}{ Medications } \\
\hline ACEI/ARB & $12(24 \%)$ & $8(28 \%)$ & 0.23 \\
\hline$\beta$-blockers & $15(30 \%)$ & $13(26 \%)$ & 0.36 \\
\hline Nitrates & $14(28 \%)$ & $15(30 \%)$ & 0.42 \\
\hline Statins & $22(44 \%)$ & $19(48 \%)$ & 0.19 \\
\hline Aspirin & $11(22 \%)$ & $8(16 \%)$ & 0.21 \\
\hline Clopidogrel & $7(14 \%)$ & $5(10 \%)$ & 0.18 \\
\hline
\end{tabular}


Fig. 1. The expression levels of HOTAIR and cTnI in AMI patients. (A) The expression levels of plasma HOTAIR in AMI patients and control subjects. (B) The expression levels of plasma cTnI in AMI patients and control subjects. (C) The expression patterns of plasma HOTAIR and cTnI in the AMI group. (D) The correlation between circulating HO-

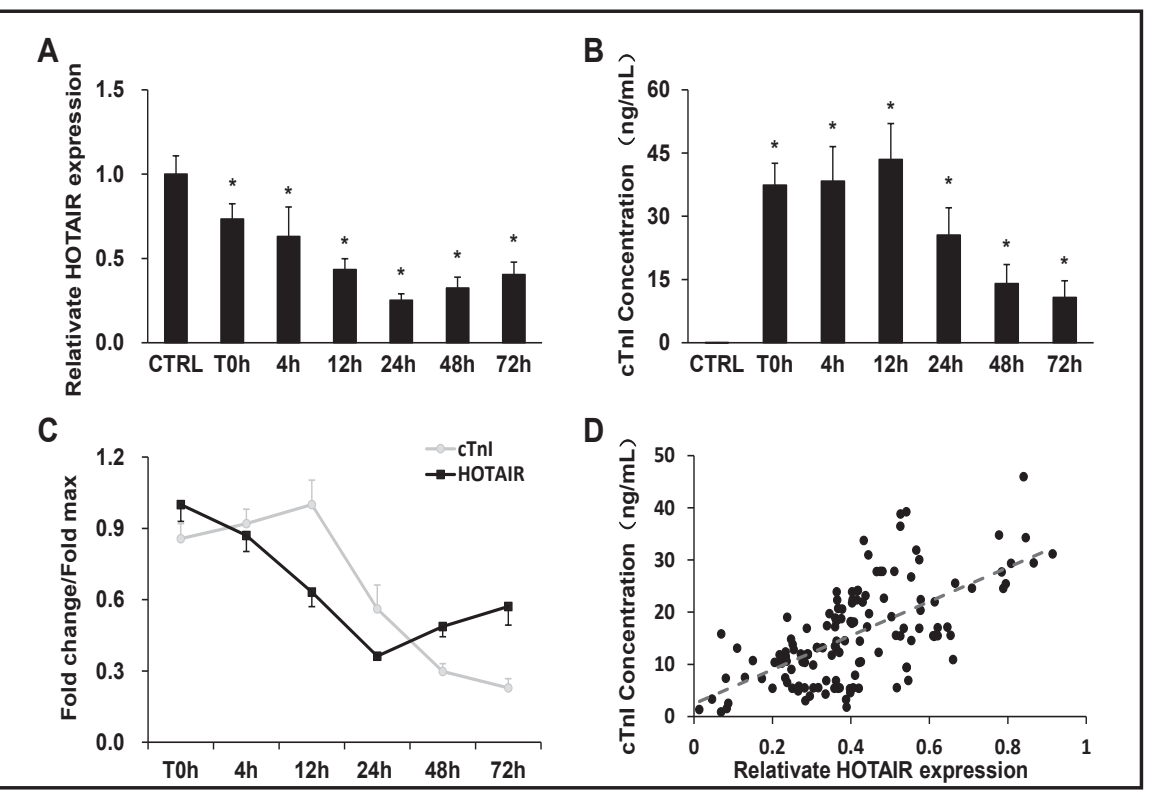
TAIR and cTnI expression levels. AMI, acute myocardial infarction; cTnI, cardiac troponin I; CTRL, controls $\left({ }^{*} \mathrm{P}<0.05\right.$ vs. control subjects).

To further determine the biological function of HOTAIR in cardiomyocytes, we transiently transfected cultured cardiomyocytes with AdHOTAIR, AdshHOTAIR, or control adenoviral vectors, and RT-PCR was used to confirm the transfection efficiency. As shown in Fig. 3B, AdHOTAIR and AdshHOTAIR significantly increased and decreased, respectively, the HOTAIR mRNA expression level in cardiomyocytes.

\section{HOTAIR regulates hypoxia-induced cardiomyocyte apoptosis in vitro}

Therefore, we evaluated the function of IncRNA HOTAIR in cardiomyocytes. To detect the effect of HOTAIR on cardiomyocyte apoptosis, we transiently transfected cultured cardiomyocytes with AdHOTAIR, AdshHOTAIR, or control adenoviral vectors; then, the cardiomyocytes were exposed to hypoxia for $6 \mathrm{~h}$. Representative photomicrographs of TUNEL-stained cultured cardiomyocytes transfected with AdHOTAIR, AdshHOTAIR, or control are shown in Fig. 4A. The results showed that compared with HOTAIR expression in the control transfection, overexpressing or silencing the expression of HOTAIR greatly inhibited or promoted hypoxia-induced apoptosis in cultured cardiomyocytes (Fig. 4B).

\section{Mechanism of HOTAIR inhibits hypoxia-induced apoptosis in cardiomyocytes}

The above results suggest that HOTAIR inhibits hypoxia-induced cardiomyocyte apoptosis in vitro. Next, we examined the signalling pathways related to hypoxia-induced apoptosis in the heart. Caspase- 3 is a key mediator of apoptosis, and its activation leads to DNA injury and subsequent apoptotic cell death. The hypoxia-induced activation and cleavage of caspase- 3 was modest in the control groups and was rarely observed in that AdHOTAIR groups; however, the activity and cleavage of caspase-3 was increased considerably when HOTAIR expression was silenced (Fig. 5A). In response to hypoxia, the expression of Bax, which functions as a proapoptotic protein, was increased, whereas the expression of the anti-apoptotic protein Bcl-2 was decreased. As predicted, HOTAIR upregulation significantly reduced the ratio of Bax to $\mathrm{Bcl}-2$, but this ratio was remarkably increased in the shHOTAIR groups exposed to hypoxia (Fig. 5B). These results indicate that the increased expression of HOTAIR protects cardiomyocytes against hypoxia-induced apoptosis. 
Fig. 2. HOTAIR is downregulated by exposure to hypoxia. (A) RTPCR analysis of HOTAIR expression in serum of C57BL/6J mice subjected to AMI and the sham operated mice for the indicated time points $(* \mathrm{P}<0.05$ vs. Sham group). (B) RT-PCR analysis of HOTAIR expression in neonatal rat cardiomyocytes exposed to hypoxia for the indicated times $\left({ }^{*} \mathrm{P}<0.05\right.$ vs. $\left.0 \mathrm{~h}\right)$.

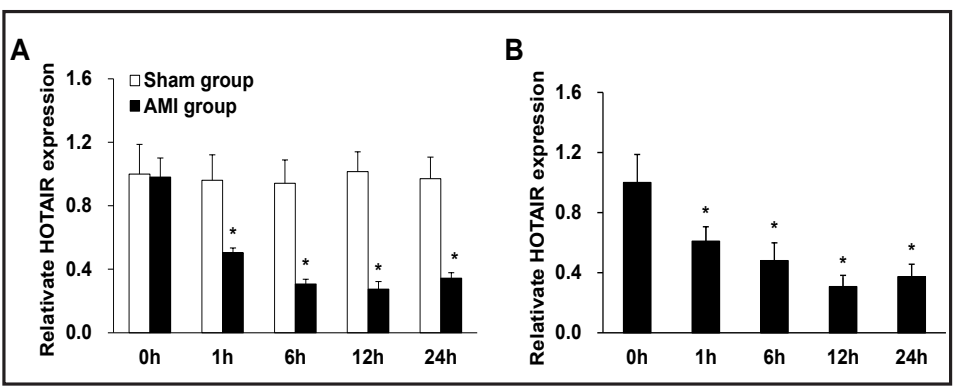

Fig. 3. HOTAIR is a myocardialspecific IncRNA. (A) RT-PCR showed the mRNA expression of HOTAIR in the rat organs, including heart, liver, spleen, lung, kidney, and muscle. (B) The mRNA expression level of HOTAIR after infection of AdHOTAIR or AdshHOTAIR ( $\left.{ }^{*} \mathrm{P}<0.05\right)$.

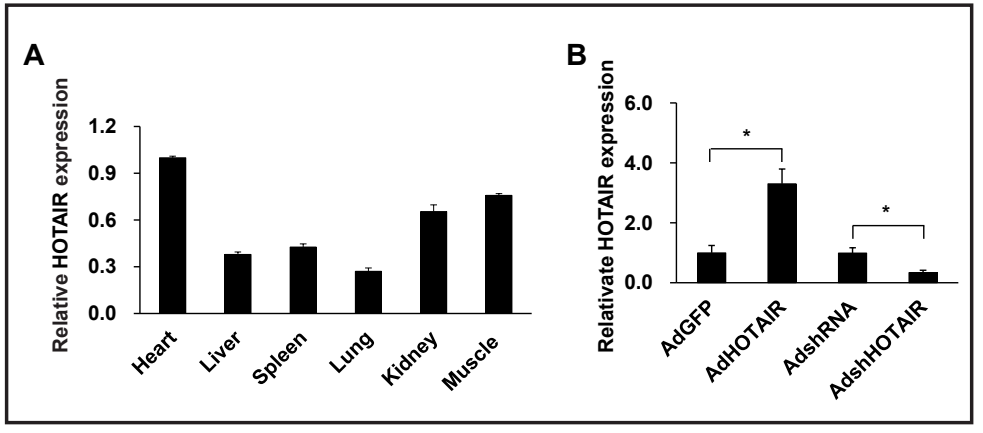

Level of circulating miR-1 in patients with AMI

Recently, reports have indicated that IncRNAs function as competing endogenous RNAs (ceRNAs) to indirectly regulate miRNA activity and hence functionally liberate other RNA transcripts. To detect whether HOTAIR has a similar mechanism in cardiomyocytes, bioinformatics analysis was performed to identify miRNA recognition sequences in HOTAIR. We found that miR-1 has a binding site on HOTAIR. To explore the potential correlation between HOTAIR and miR-1 in patients with AMI, we examined the expression level of miR1 in AMI patients. As shown in Fig. 6A, the expression level of miR-1 in all AMI patients was significantly increased in the early phase (the first 3 time points) following the occurrence of AMI compared with that of the healthy volunteers. Furthermore, Pearson correlation analysis confirmed that the HOTAIR expression level was negatively related to miR-1 expression in AMI patients (Fig. 6B).

\section{HOTAIR serves as a miR-1 sponge}

As shown in Fig. 7A, the bioinformatics analysis found that miR-1 had a binding site on HOTAIR. To confirm the direct binding relationship between HOTAIR and miR-1, a luciferase activity assay was performed. The results showed that co-transfection of miR- 1 with CMV promoter HOTAIR-WT significantly decreased luciferase activity, whereas co-transfection of miR-1 with CMV promoter HOTAIR-MUT did not change the luciferase activity (Fig. 7B). In addition, we found a negative correlation between the mRNA expression level of HOTAIR and miR-1, compared with the expression in control groups, miR-1 expression was decreased in AdHOTAIR transfected cells, whereas transfection with shHOTAIR upregulated miR1 expression (Fig. 7C). Moreover, our study found that after overexpression of miR-1, the expression levels of HOTAIR were significantly downregulated compared to those in the NC (transfected with plasmid of random sequences) groups (Fig. 7D).

\section{Discussion}

AMI is one of the major causes of mortality and morbidity in the world; the heart of patients with AMI cannot continue to function without adequate blood flow, and if blood 


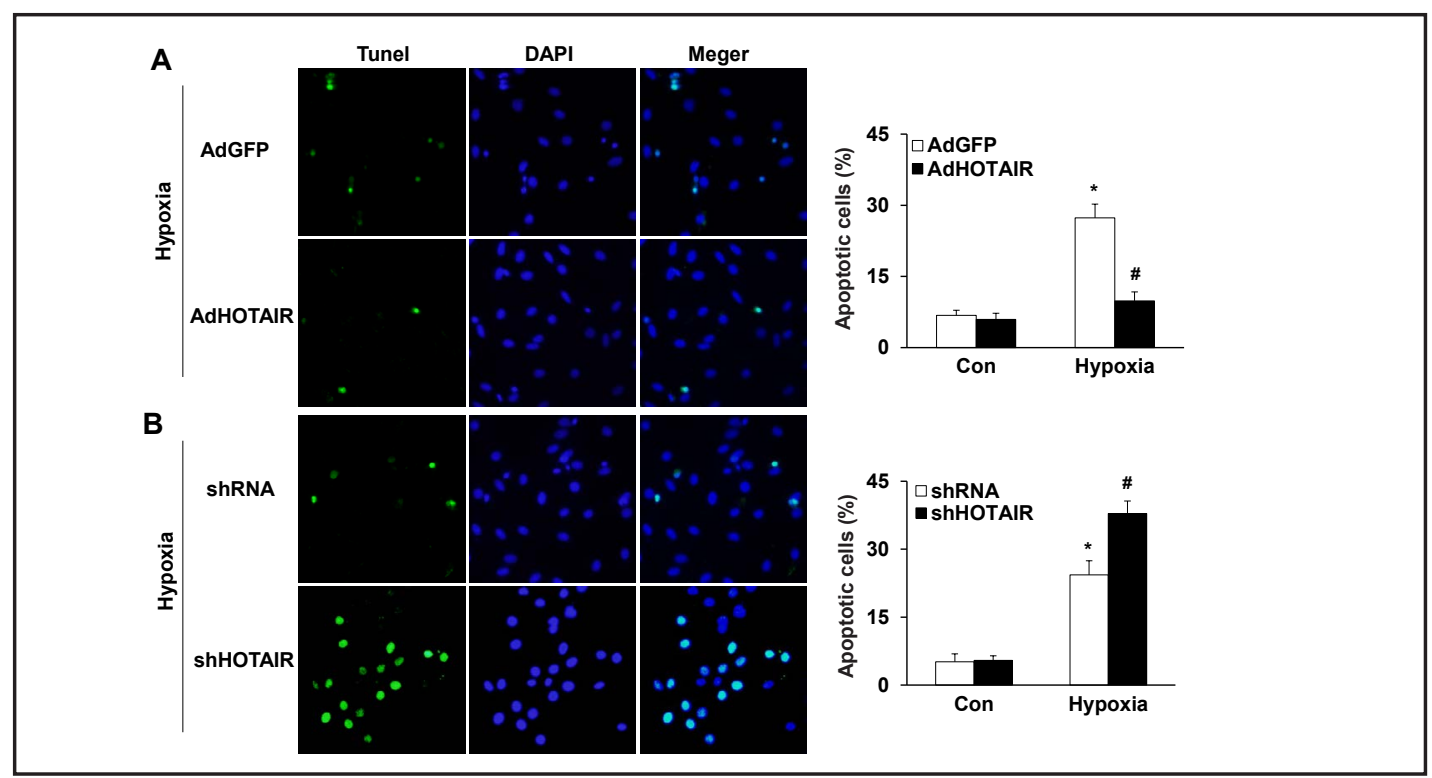

Fig. 4. Effects of HOTAIR on apoptosis in cardiomyocytes. (A) Representative and quantitative results of TUNEL staining in the AdGFP and AdHOTAIR group exposed to hypoxia ( ${ }^{*} \mathrm{P}<0.05$ vs. AdGFP). (B) Representative and quantitative results of TUNEL staining in the AdshRNA and AdshHOTAIR groups exposed to hypoxia ( ${ }^{*} \mathrm{P}<0.05$ vs. AdshRNA). TUNEL, terminal deoxynucleotide transferase dUTP nick end labelling.

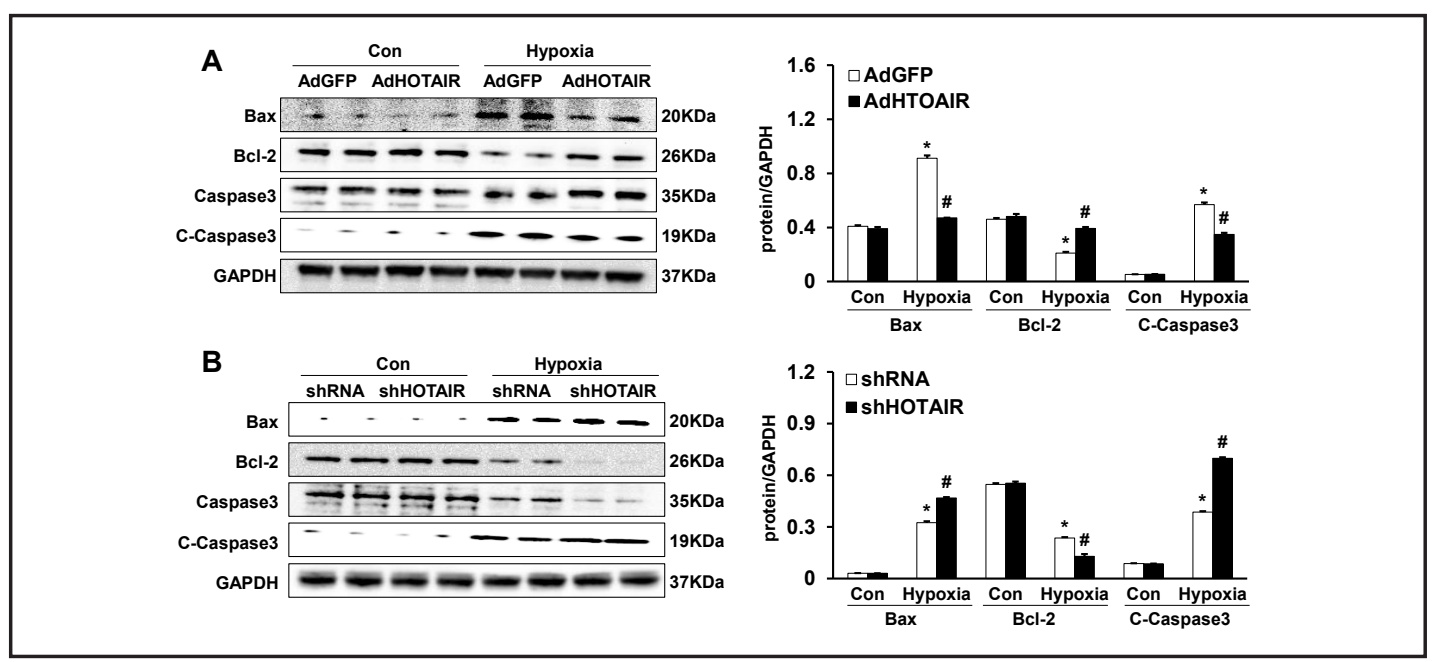

Fig. 5. Mechanism of HOTAIR inhibits hypoxia-induced apoptosis in cardiomyocytes. (A) Western blot analysis of Bax, Bcl-2, and caspase-3 protein expression in cardiomyocytes from the AdGFP and AdHOTAIR groups after normoxic or hypoxic exposure $\left({ }^{*} \mathrm{P}<0.05\right.$ vs. AdGFP /normoxic; ${ }^{\#} \mathrm{P}<0.05$ vs. AdGFP /hypoxia). (B) Western blot analysis of Bax, Bcl-2, and caspase-3 protein expression in cardiomyocytes from the AdshRNA and AdshHOTAIR groups after normoxic or hypoxic exposure ( ${ }^{*} \mathrm{P}<0.05$ vs AdshRNA /normoxic; ${ }^{\#} \mathrm{P}<0.05$ vs AdshRNA / hypoxia).

flow is severely compromised, death is inevitable [1, 2]. Therefore, specific and early-stage biomarkers that can be used clinically to establish a timely diagnosis in patients with AMI are increasingly important. In this study, we demonstrated that the expression of HOTAIR is significantly downregulated in the early phase of acute myocardial infarction. Furthermore, our data demonstrate for the first time a direct correlation between the expression level of HOTAIR and the expression of miR-1 in AMI. 
Fig. 6. The expression level of miR-1 and the relationship between HOTAIR and miR1 in AMI patients. (A) The expression level of miR-1 in AMI patients. $\left({ }^{*} \mathrm{P}<0.05\right.$ vs. CTRL). (B) Plot analysis of the relationship between HOTAIR and miR-1 in AMI patients.

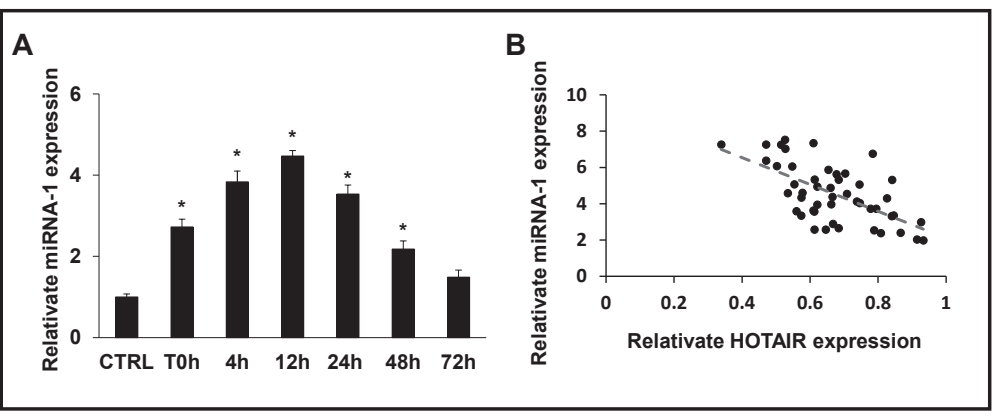

A

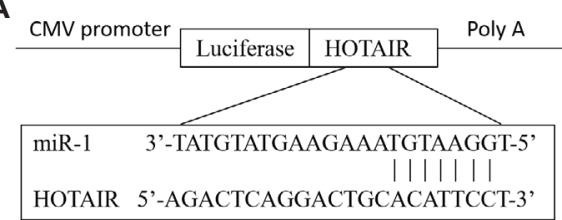

C

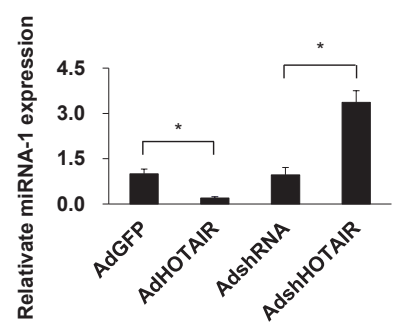

B

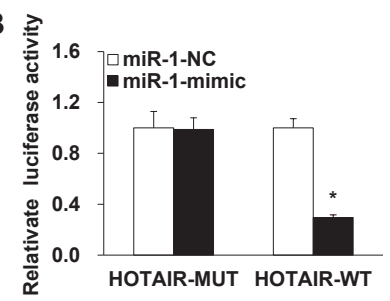

D

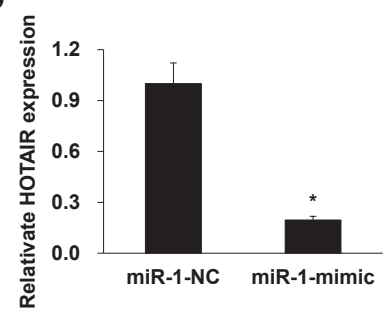

Fig. 7. HOTAIR functionally interacted with miR-1. (A) The HOTAIR transcript contains putative miRNA recognition sites complementary to miR-1. (B) Luciferase reporter plasmids containing HOTAIR-WT or HOTAIR-Mut were transfected into cardiomyocytes together with miR-1 in parallel with a miR-NC plasmid vector ( ${ }^{*} \mathrm{P}<0.05$ vs. WT/NC group). (C) The expression level of miR-1 after infection with AdHOTAIR or AdshHOTAIR. (D) The expression level of HOTAIR in neonatal rat cardiomyocytes after treatment with miRNC or miR-1-mimic ( ${ }^{*} \mathrm{P}<0.05$ vs. NC group).

LncRNAs are a type of RNA with a length of approximately 200 nucleotides that are not translated. LncRNAs are stable in plasma and show disease and tissue specificity. To date, more than 1000 lncRNAs have been confirmed to be involved in various biological processes, including cell growth, differentiation, cell proliferation and apoptosis [10-13]. An increasing number of studies have suggested that circulating plasma lncRNAs have great potential as new diagnostic and prognostic biomarkers and contribute to the effective evaluation of treatment in diseases such as cardiovascular disease and cancer. Recently, an increasing number of circulating lncRNAs, including heart-, vascular- and muscle-specific lncRNAs, have been reported as new biomarkers for multiple cardiovascular diseases [14]. Vausort and colleagues identified 5 lncRNAs, and the expression levels of these lncRNAs in blood were tremendously altered after MI, which may help to predict the outcomes for AMI patients [29]. The plasma concentration of the lncRNA CoroMarker was recently recognized as a novel biomarker for the diagnosis of coronary artery disease due to the stability, sensitivity and specific properties of CoroMarker in these patients [30]. The circulating concentration of the lncRNA UCA1 has also been identified as a potential novel biomarker for the prognosis of AMI [31]. These observations support the potential for lncRNAs to be used as biomarkers and therapeutics in ischaemic heart injury, especially AMI.

The HOTAIR IncRNA was introduced as a spliced and polyadenylated RNA with 2, $158 \mathrm{nt}$ and six exons [21]. This RNA originates from the transcription of the antisense strand of the 
HOXC gene, which is located between HOXC11 and HOXC12 on chromosome 12q13.13. As one of the first identified lncRNAs, HOTAIR has been verified as an important modulator in the progression of different kinds of cancer $[19,20]$. Recently, studies have suggested that the human HOTAIR IncRNA functions as a ceRNA in ovarian and gastric carcinogenesis [32, 33]. Moreover, HOTAIR was reported as being significantly upregulated in the cardiomyocytes of mice with lipopolysaccharide-induced sepsis [23]. Lai et al. found that HOTAIR plays an important role in inhibiting the progress of cardiac hypertrophy [24]. In addition, studies have shown that high expression levels of HOTAIR promote the onset of hypoxia-induced ischaemic infarcts [25]. Altogether, the above observations support at crucial role for HOTAIR in cardiovascular diseases. However, whether HOTAIR is involved in the regulation of AMI and can serve as a biomarker for AMI is still unknown.

Our results demonstrated that in AMI patients the expression levels of HOTAIR were significantly decreased in the early phase following the occurrence of AMI, were lowest from 6-12 $\mathrm{h}$ after AMI occurred, and exhibited an opposite trend as cTnI. In addition, the results showed a negative correlation between the mRNA level of HOTAIR and cTnI. Next, we quantified the expression level of HOTAIR in the serum of AMI mice and in cultured cardiomyocytes exposed to hypoxia; quantitative RT-PCR demonstrated that the expression level of HOTAIR was remarkably reduced in both the the serum of AMI mice and the cultured cardiomyocytes exposed to hypoxia. These data strongly support the possibility that HOTAIR may serve as a biomarker for AMI diagnosis.

Recent evidence has demonstrated that a sudden increase in cardiomyocyte apoptosis occurs in the ischaemic hearts of both patient and animals, and apoptosis leads to continuous cardiomyocyte loss throughout the entire post-MI pathological process. Moreover, inhibiting cardiomyocyte apoptosis has shown potential protection against post-MI cardiac dysfunction [34, 35]. The experimental findings herein demonstrated that HOTAIR mediates cardiomyocyte apoptosis: the overexpression of HOTAIR significantly reduced the number of apoptotic cells, whereas HOTAIR depletion increased the number of apoptotic cells. Additionally, the imbalance between pro-apoptotic proteins (such as Bax and cleaved caspase-3) and anti-apoptotic proteins (Bcl-2) has long been established as a key determinant in myocardial apoptosis. In support of the anti-apoptotic function of HOTAIR, we observed that HOTAIR downregulated the protein expression of both Bax and cleaved caspase-3, but increased the expression level of Bcl-2. Taken together, these results indicate that HOTAIR protects against cardiac dysfunction after MI by inhibiting cell death through the regulation of apoptosis-related proteins. In fact, apoptosis-induced cardiomyocyte loss is a common pathological process shared by many cardiomyopathy-related disorders. Thus, our studies provide clues about the role of HOTAIR in other types of apoptosis-related cardiomyopathies, such as dilated cardiomyopathy and peripartum cardiomyopathy.

Previous studies have established that lncRNAs can act as ceRNAs to sponge miRNAs, consequently relieving the suppression of miRNA targets [36, 37]. In this study, miR-1 expression was found to be markedly increased in AMI patients compared with that of the controls, and we also found that HOTAIR expression was negatively correlated with the expression of miR-1. Overexpression of HOTAIR downregulated the miR-1 expression level, whereas knockdown HOTAIR induced the upregulation of miR-1 expression. By contrast, overexpression of miR-1 downregulated the HOTAIR expression level. Thus, HOTAIR and miR-1 may form a reciprocal repression feedback loop. The mechanism of this feedback loop was explored in the present study, and it was observed that miR-1 regulates the expression of HOTAIR by directly binding to target sites within the HOTAIR sequence. This is consistent with results in hepatocellular carcinoma [38]. All these data suggest that HOTAIR can bind miR-1 and subsequently contributes to the regulation of AMI.

\section{Conclusion}

In summary, the present study measured the early changes of circulating HOTAIR in AMI patients and provided novel insights into the relationship between plasma HOTAIR and miR- 
1 in AMI patients; our results suggested that circulating HOTAIR may serve as a sensitive predictor for diagnosing AMI. This study not only provides new insights into HOTAIR for the diagnosis of AMI but also furthers the understanding of IncRNA function in AMI pathogenesis.

\section{Acknowledgements}

This study was supported by the National Natural Science Foundation of China (Grant Nos. 81600191 and 81600189), the Medical Science and Technology Research Project of Henan province (Grant No. 201702063) and the Scientific and Technological Project of Henan province (Grant No. 172102310531).

\section{Disclosure Statement}

The authors declare that no conflict of interest exists.

\section{References}

1 Sacks NC, Ash AS, Ghosh K, Rosen AK, Wong JB, Rosen AB: Trends in acute myocardial infarction hospitalizations: Are we seeing the whole picture? Am Heart J 2015;170:1211-1219.

2 Nawaz W, Khan FU, Khan MZ, Gang W, Yang M, Liao X, Zhang L, Ihsan AU, Khan A, Han L, Zhou X: Exoorganoplasty interventions: A brief review of past, present and future directions for advance heart failure management. Biomed Pharmacother 2017;88:162-172.

-3 White HD, Chew DP: Acute myocardial infarction. Lancet 2008;372:570-584.

4 Jones BM, Kapadia SR, Smedira NG, Robich M, Tuzcu EM, Menon V, Krishnaswamy A: Ventricular septal rupture complicating acute myocardial infarction: a contemporary review. Eur Heart J 2014;35:2060-2068.

5 Greco S, Gorospe M, Martelli F: Noncoding RNA in age-related cardiovascular diseases. J Mol Cell Cardiol 2015;83:142-155.

6 van Rooij E, Olson EN: MicroRNA therapeutics for cardiovascular disease: opportunities and obstacles. Nat Rev Drug Discov 2012;11:860-872.

7 Almeida MI, Reis RM, Calin GA: MicroRNA history: discovery, recent applications, and next frontiers. Mutat Res 2011;717:1-8.

-8 Da Sacco L, Baldassarre A, Masotti A: Bioinformatics tools and novel challenges in long non-coding RNAs (lncRNAs) functional analysis. Int J Mol Sci 2012;13:97-114.

-9 Pang L, Hu J, Zhang G, Li X, Zhang X, Yu F, Lan Y, Xu J, Pang B, Han D, Xiao Y, Li X: Dysregulated long intergenic non-coding RNA modules contribute to heart failure. Oncotarget 2016;7:59676-59690.

10 Geisler S, Coller J: RNA in unexpected places: long non-coding RNA functions in diverse cellular contexts. Nat Rev Mol Cell Biol 2013;14:699-712.

11 Roberts TC, Morris KV, Weinberg MS: Perspectives on the mechanism of transcriptional regulation by long non-coding RNAs. Epigenetics 2014;9:13-20.

12 Fatica A, Bozzoni I: Long non-coding RNAs: new players in cell differentiation and development. Nat Rev Genet 2014;15:7-21.

13 Li C, Chen J, Zhang K, Feng B, Wang R, Chen L: Progress and Prospects of Long Noncoding RNAs (lncRNAs) in Hepatocellular Carcinoma. Cell Physiol Biochem 2015;36:423-434.

14 Kumarswamy R, Bauters C, Volkmann I, Maury F, Fetisch J, Holzmann A, Lemesle G, de Groote P, Pinet F, Thum T: Circulating long noncoding RNA, LIPCAR, predicts survival in patients with heart failure. Circ Res 2014;114:1569-1575.

15 Xuan L, Sun L, Zhang Y, Huang Y, Hou Y, Li Q Guo Y, Feng B, Cui L, Wang X, Wang Z, Tian Y, Yu B, Wang S, Xu C, Zhang M, Du Z, Lu Y, Yang BF: Circulating long non-coding RNAs NRON and MHRT as novel predictive biomarkers of heart failure. J Cell Mol Med 2017;21:1803-1814.

16 Kitow J, Derda AA, Beermann J, Kumarswarmy R, Pfanne A, Fendrich J, Lorenzen JM, Xiao K, Bavendiek U, Bauersachs J, Thum T: Mitochondrial long noncoding RNAs as blood based biomarkers for cardiac remodeling in patients with hypertrophic cardiomyopathy. Am J Physiol Heart Circ Physiol 2016;311:H707712. 


\section{Cellular Physiology Cell Physiol Biochem 2017;44:1497-1508 \begin{tabular}{ll|l} 
and Biochemistry Published onlIne: December 01, 2017 & $\begin{array}{l}\text { (c) } 2017 \text { The Author(s). Published by S. Karger AG, Basel } \\
\text { www.karger.com/cpb }\end{array}$
\end{tabular}}

17 Wang K, Liu F, Zhou LY, Long B, Yuan SM, Wang Y, Liu CY, Sun T, Zhang XJ, Li PF: The long noncoding RNA CHRF regulates cardiac hypertrophy by targeting miR-489 C Circ Res 2014;114:1377-1388.

18 Chen J, Lin C, Yong W, Ye Y, Huang Z: Calycosin and genistein induce apoptosis by inactivation of HOTAIR/pAkt signaling pathway in human breast cancer MCF-7 cells. Cell Physiol Biochem 2015;35:722-728.

19 Bhan A, Mandal SS: LncRNA HOTAIR: A master regulator of chromatin dynamics and cancer. Biochim Biophys Acta 2015;1856:151-164.

-20 Xu CZ, Jiang C, Wu Q Liu L, Yan X, Shi R: A Feed-Forward Regulatory Loop between HuR and the Long Noncoding RNA HOTAIR Promotes Head and Neck Squamous Cell Carcinoma Progression and Metastasis. Cell Physiol Biochem 2016;40:1039-1051.

-21 Rinn JL, Kertesz M, Wang JK, Squazzo SL, Xu X, Brugmann SA, Goodnough LH, Helms JA, Farnham PJ, Segal E, Chang HY: Functional demarcation of active and silent chromatin domains in human HOX loci by noncoding RNAs. Cell 2007;129:1311-1323.

22 Song J, Kim D, Han J, Kim Y, Lee M, Jin EJ: PBMC and exosome-derived Hotair is a critical regulator and potent marker for rheumatoid arthritis. Clin Exp Med 2015;15:121-126.

23 Wu H, Liu J, Li W, Liu G, Li Z: LncRNA-HOTAIR promotes TNF-alpha production in cardiomyocytes of LPSinduced sepsis mice by activating NF-kappaB pathway. Biochem Biophys Res Commun 2016;471:240-246.

24 Lai Y, He S, Ma L, Lin H, Ren B, Ma J, Zhu X, Zhuang S: HOTAIR functions as a competing endogenous RNA to regulate PTEN expression by inhibiting miR-19 in cardiac hypertrophy. Mol Cell Biochem 2017;432:179187.

-25 Yang L, Lu ZN: Long non-coding RNA HOTAIR promotes ischemic infarct induced by hypoxia through upregulating the expression of NOX2 Biochem Biophys Res Commun 2016;479:186-191.

-26 Greco S, Zaccagnini G, Perfetti A, Fuschi P, Valaperta R, Voellenkle C, Castelvecchio S, Gaetano C, Finato N, Beltrami AP, Menicanti L, Martelli F: Long noncoding RNA dysregulation in ischemic heart failure. J Transl Med 2016;14:183.

-27 Li HL, Zhuo ML, Wang D, Wang AB, Cai H, Sun LH, Yang Q, Huang Y, Wei YS, Liu PP, Liu DP, Liang CC: Targeted cardiac overexpression of A20 improves left ventricular performance and reduces compensatory hypertrophy after myocardial infarction. Circulation 2007;115:1885-1894.

28 Gao L, Huang K, Jiang DS, Liu X, Huang D, Li H, Zhang XD, Huang K: Novel role for caspase-activated DNase in the regulation of pathological cardiac hypertrophy. Hypertension 2015;65:871-881.

29 Vausort M, Wagner DR, Devaux Y: Long noncoding RNAs in patients with acute myocardial infarction. Circ Res 2014;115:668-677.

-30 Yang Y, Cai Y, Wu G, Chen X, Liu Y, Wang X, Yu J, Li C, Chen X, Jose PA, Zhou L, Zeng C: Plasma long noncoding RNA, CoroMarker, a novel biomarker for diagnosis of coronary artery disease. Clin Sci (Lond) 2015;129:675-685.

31 Yan Y, Zhang B, Liu N, Qi C, Xiao Y, Tian X, Li T, Liu B: Circulating Long Noncoding RNA UCA1 as a Novel Biomarker of Acute Myocardial Infarction. Biomed Res Int 2016;2016:8079372.

-32 Kim K, Jutooru I, Chadalapaka G, Johnson G, Frank J, Burghardt R, Kim S, Safe S: HOTAIR is a negative prognostic factor and exhibits pro-oncogenic activity in pancreatic cancer. Oncogene 2013;32:1616-1625.

33 Gupta RA, Shah N, Wang KC, Kim J, Horlings HM, Wong DJ, Tsai MC, Hung T, Argani P, Rinn JL, Wang Y, Brzoska P, Kong B, Li R, West RB, van de Vijver MJ, Sukumar S, Chang HY: Long non-coding RNA HOTAIR reprograms chromatin state to promote cancer metastasis. Nature 2010;464:1071-1076.

34 Wan N, Liu X, Zhang XJ, Zhao Y, Hu G, Wan F, Zhang R, Zhu X, Xia H, Li H: Toll-interacting protein contributes to mortality following myocardial infarction through promoting inflammation and apoptosis. Br J Pharmacol 2015;172:3383-3396.

35 Liu X, Wan N, Zhang XJ, Zhao Y, Zhang Y, Hu G, Wan F, Zhang R, Zhu X, Xia H, Li H: Vinexin-beta exacerbates cardiac dysfunction post-myocardial infarction via mediating apoptotic and inflammatory responses. Clin Sci (Lond) 2015;128:923-936.

-36 Mattick JS, Makunin IV: Non-coding RNA. Hum Mol Genet 2006;15 Spec No 1:R17-29.

-37 Gibb EA, Brown CJ, Lam WL: The functional role of long non-coding RNA in human carcinomas. Mol Cancer 2011;10:38.

-38 Su DN, Wu SP, Chen HT, He JH: HOTAIR, a long non-coding RNA driver of malignancy whose expression is activated by FOXC1, negatively regulates miRNA-1 in hepatocellular carcinoma. Oncol Lett 2016;12:40614067. 